\title{
Magnetic field reduction by means of active shielding techniques
}

\author{
S. Celozzi \& F. Garzia \\ Department of Electrical Engineering \\ University of Rome "La Sapienza", Italy
}

\begin{abstract}
Different techniques for the reduction of the magnetic field arising from either overhead lines or buried cables are presented, based on the use of active conductors for the compensation.

The optimal choice of the currents to be driven in the compensation wires is attained by means of a genetic algorithm, which compares favourably with deterministic algorithms in terms of accuracy and computational costs.

The procedure may be applied to any kind of source configuration, with appropriate modifications, ensuring significant magnetic field reductions.
\end{abstract}

\section{Introduction}

Increasing attention is actually devoted to the magnetic field levels due to power lines either in the overhead and cable configuration. Various mitigation strategies are available and are generally applied and experienced at the design stage, while the possibilities of achieving a real reduction strongly reduce when existing installations are dealt with.

One of the most attractive technologies applicable to already realized power lines relies on the so-called active shielding [1], which consists in the design of antagonist sources capable of generating, in a given region, a magnetic field almost opposite to that of the original source. Such a compensation can be achieved only in the average sense, however a considerable reduction is generally possible at reasonable costs.

It should be noted, in fact, that passive shielding technologies based on the use of panels realized by means of conductive and/or ferromagnetic are often not feasible and generally present poor performance. Moreover, the costs 


\section{Environmental Health Risk}

associated with works of reconfiguration of existing power lines are generally very high.

In this work an overview of a general and efficient procedure for the evaluation of the optimal currents to be driven in the auxiliary compensation conductors is presented. The procedure is based on a genetic algorithm which, starting from the source configuration data (electrical and geometrical) searches in user-provided intervals the optimal configuration of the new conductors to be laid and driven. In case of high-voltage overhead lines, one of the active shielding conductors may be the existing guard-wire.

It will be shown that very often two additional conductors (one if the guard wire is used for this purpose) are necessary for achieving a strong reduction; the realization aspects will take eventually advantage from the possibility of using two currents opposite in phase.

Examples are presented with reference to either the cable and the overhead configuration and demonstrate how reductions on the magnetic field levels in the order of $70 \%-80 \%$ are easily achievable.

\section{The active shielding}

New limits for human exposure to low-frequency magnetic field generated by power lines issued in several countries call for the search of solutions for the mitigation of the root mean square (r. m. s.) value of the magnetic flux density.

In Figures 1 two typical configurations requiring a mitigation are sketched, with reference to overhead three-phase or buried cable transmission line. An active shielding solution is proposed for the reduction of the magnetic flux density in the grey area below a threshold value: a current is driven in one or more additional conductors, one of them being the guard-wire if existing in order to reduce the installation costs. In the following, it has been considered that the two compensation currents are opposite in phase, avoiding in this way their return through the earth, altough other solutions may be adopted and work quite well.

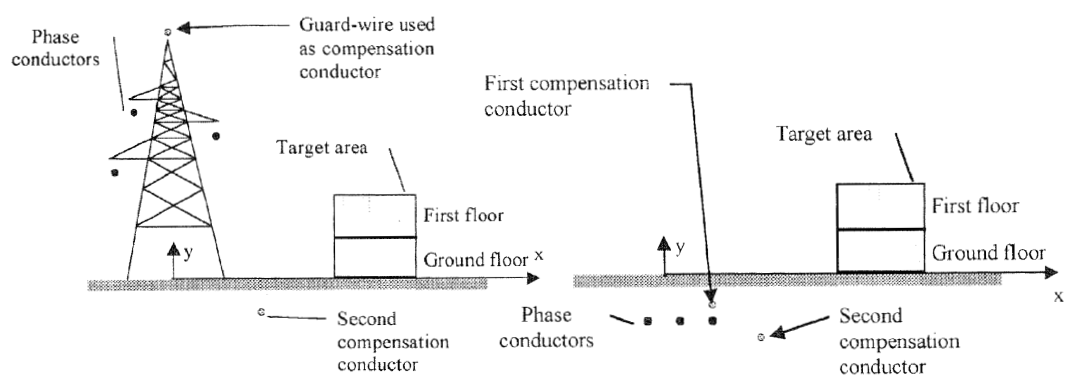

(a)

(b)

Figure 1 - Three-phase overhead line (a) and buried cable line (b) to be actively shielded. 
Input data are: coordinates of the phase conductors and their currents (rms values and phases), position of the guard-wire (if any), region of interest, maximum admissible magnetic flux density in the target area. Output data will be: number, position and values of the currents in the compensation wires. Constraints are the intervals of admissible positions for the compensation wires, values of their currents. The genetic algorithm adopted to solve this optimization problem is described in the following section, after having introduced three main assumptions which are at the basis of the shielding design procedure:

1) the magnetic flux density is assumed to have only $x$ - and $y$ components,

2) the conductors are parallel to the ground,

3) the area where the mitigation is requested is located on one side only with respect to the line.

The first assumption is reasonably verified in absence of changes of line direction, whereas the second is not essential and may be easily removed by inserting into the formulation the effects of the conductor sags. The last hypothesis is often verified and its removal implies some modifications in the design procedure. Both overhead lines and buried cables may be considered as magnetic field sources and balanced or unbalanced three-phase systems are admissible as well.

\section{The genetic algorithms optimisation technique}

Genetic algorithms [GAs] are wide-range numerical optimisation methods, based on the use of natural processes of evolution and genetic recombination [5]. Thanks to their versatility, they can be applied different fields, and they also find a lot of applications in electromagnetic optimization problems [2-4].

GAs are particularly useful when the goal is to find an approximate global minimum in a high-dimension, multi-modal function domain, in a near-optimal manner. The algorithms encode each parameters of the problem to be optimised into a proper sequence (where the alphabet used is generally binary), called gene, and combine the different genes to constitute a chromosome. A proper set of chromosomes, called population, undergoes the Darwinian processes of natural selection, mating and mutation, creating new generations, until it reaches the final optimal solution. GAs show their power in active shielding problems, where different parameters, such as position of active conductors and current that flows inside them, must be optimised to reduce the magnetic field in a given target area.

\section{Reduction of magnetic field produced by overhead lines}

The reduction of magnetic field produced by overhead lines is obtained, as explained before, using proper currents that circulate in the guard wire and in the underground compensation cable, whose position is properly searched to obtaine the maximum reduction.

The magnetic flux density distribution $\mathrm{B}(\mathrm{x}, \mathrm{y})$ in the considered target area strictly depends on the power line geometry and the related currents on the 


\section{Environmental Health Risk}

dimensions of the target area, and on the relative distance between the power line and the considered target area. For this reason we expect to obtain different results in term of percentage of reduction of $\mathrm{B}$ and in terms of position and current in the compensation wires when one of the mentioned parameter varies.

We mainly focus our attention on a fixed power line geometry and various situations for the target area have been considered.

The power line geometrical arrangement is reported in Table I. It has been considered a power line current I whose magnitude is equal to $300 \mathrm{~A}$ in each phase, even if the considered GA algorithm expresses the compensation currents as ratio with respect to the power line current, ensuring the obtained reduction solutions to be invariable with respect to variations of magnitude of power line current (if the compensation currents follow these variations according to the mentioned ratios).

A target area whose height is equal to 10 meters and whose width is equal to 10 meters has been considered. The relative distance power line-target area has been varied and the magnetic field reduction has been optimised by the GA, obtaining the results reported in Table II and shown in figs.2.

The GA has obviously calculated different solutions for each considered geometry (due to the different individuals that compose the evolving population), but only the solution characterized by the higher percentage of reduction of the magnetic flux density has been considered for each case.

Table I Geometrical arrangement of the considered power line

\begin{tabular}{|l|l|l|l|}
\hline Wire & $x[\mathrm{~m}]$ & $y[\mathrm{~m}]$ & Current $[\mathrm{A}]$ \\
\hline$\# 1$ & -3 & 30 & $900 \angle 0^{\circ}$ \\
\hline$\# 2$ & 0 & 30 & $900 \angle-120^{\circ}$ \\
\hline$\# 3$ & 3 & 30 & $900 \angle-240^{\circ}$ \\
\hline$\# 4$ (guard wire) & 0 & 33 & To be optimised \\
\hline
\end{tabular}


Table II Results of the optimisation

\begin{tabular}{|c|c|c|c|c|c|c|c|}
\hline \multirow[b]{2}{*}{ 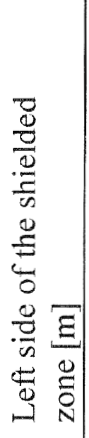 } & \multirow[b]{2}{*}{ 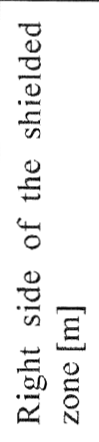 } & \multirow[b]{2}{*}{ 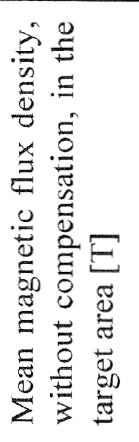 } & \multicolumn{5}{|c|}{ Guard wire + underground cable reduction based } \\
\hline & & & 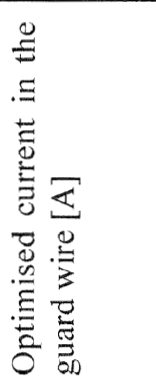 & & 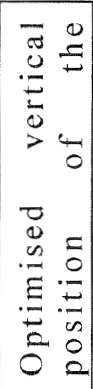 & 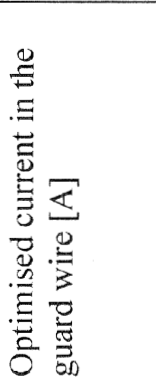 & 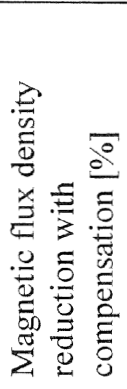 \\
\hline & +10 & $1.50 \cdot 10^{-6}$ & $27 \angle 5^{\circ}$ & -40 & -1.6 & 2794 & 67.96 \\
\hline & +20 & $1.15 \cdot 10^{-6}$ & $153 \angle 33$ & -22 & -1.1 & 1802 & 80.78 \\
\hline & +30 & $7.83 \cdot 10^{-7}$ & $144 \angle 329^{\circ}$ & +2 & -2.5 & $0321+3$ & 92.10 \\
\hline & +40 & $5.27 \cdot 10^{-7}$ & $153 \angle 329^{\circ}$ & & -3.1 & $126 \angle 147^{\circ}$ & 93.19 \\
\hline
\end{tabular}

Different considerations can be drawn from the obtained results.

The first consideration is that the proposed compensation method, applied to this particular power line arrangement, ensures significant reductions of the mean magnetic flux density that varies with the relative distance power linetarget area, as it was expected, reaching values of the order of $93 \%$ and that are never lower than $68 \%$.

The second consideration is that the underground cable is always placed on the same side of the power line with respect to the target area. The position of the underground cable varies obviously with the relative distance power linetarget area to reduce, as much as possible, the mean value of the magnetic flux density in the target area itself.

The third consideration is that the compensated magnetic flux density distribution inside the target area tends to assume a radial symmetry with respect to the approximate centre of the considered area instead of a radial symmetry with respect to the power line.

The fourth consideration is that the obtained values of the current inside the guard wire tend to be very similar in phase and variable in amplitude.

The last consideration is that the obtained values of the current inside the underground cable tend to show the same behaviour of the guard wire.

To verify the validity of the proposed method, different power lines geometries and different target area configurations were optimised by the GA, obtaining generally mean magnetic flux density reduction greater than $50 \%$.

As an example, it is considered a power line characterized by a reduced height with respect to the ground and by a wider distance between the conductors that generate a more intense and differently space distributed magnetic flux density in the target area. 


\section{Environmental Health Risk}

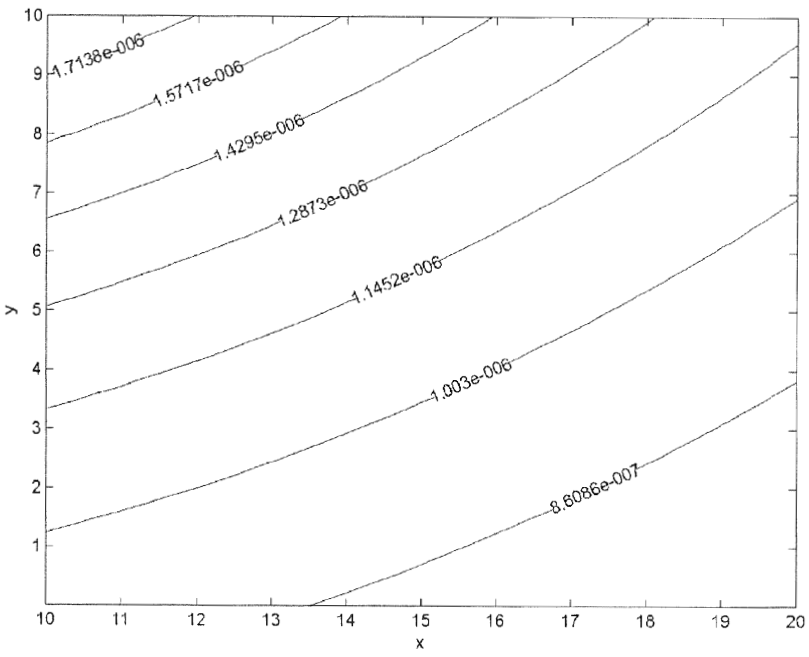

Fig.2a

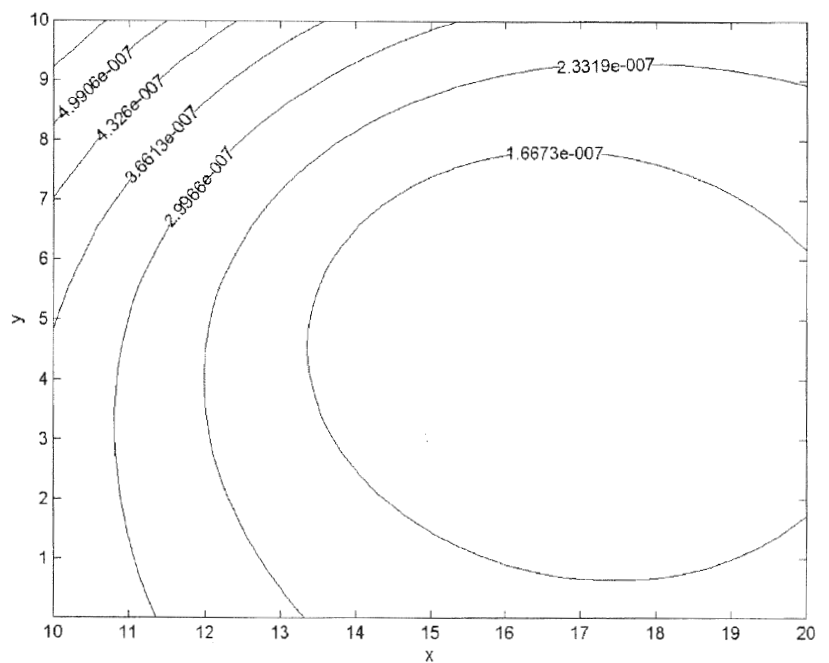

Fig. $2 b$

Figs.2 Magnetic flux density [T] in the considered section, (height=10 $\mathrm{m}$ ) positioned between $+10 \mathrm{~m}$ and $+20 \mathrm{~m}$ with respect to the power line vertical axes for different situations.

a) Magnetic field in the absence of compensation (mean magnetic flux density $=1.15 \cdot 10^{-6} \mathrm{~T}$ ).

b) (Guard wire + underground cable) - based compensation: magnetic field in the presence of a compensation current equal to $153 \angle 335^{\circ} \mathrm{A}$ in the guard wire positioned in $(0,33)$ and a compensation current equal to $180 \angle 152^{\circ} \mathrm{A}$ in the underground cable positioned in $(-22,-1.1)$. The mean magnetic flux density is $80.78 \%$ lower than that in the uncompensated situation. 
Even in this case a significant reduction (about $60 \%$ ) of the mean magnetic flux density in the target area is obtained, demonstrating the validity of the proposed technique.

\section{Reduction of magnetic field produced by buried cables}

The reduction of magnetic field produced by underground cables is obtained, as explained before, using proper currents that circulate in one or two underground cables, whose position is properly searched to obtaine the maximum reduction. In the case of two underground cables the current that flows inside them is equal in amplitude and opposite in phase.

The magnetic flux density distribution $\mathrm{B}(\mathrm{x}, \mathrm{y})$ in the considered target area strictly depends on the underground cable geometry and the related currents, on the dimensions of the target area, and on the relative distance between the underground cable and the considered target area. For this reason we expect to obtain different results in terms of percentage of reduction of $B$ and in terms of position and current in the compensation cables when one of the mentioned parameter varies.

We mainly focus our attention on a fixed underground cable geometry and various situations for the target area have been considered.

The geometrical arrangement of the underground cable is reported in Table III. It has been considered an underground cable current I whose magnitude is equal to $450 \mathrm{~A}$ in each phase, even if the considered GA algorithm expresses the compensation currents as ratio with respect to the underground cable current, ensuring the obtained reduction solutions to be invariable with respect to variations of magnitude of the underground cable current (if the compensation currents follow these variations according to the mentioned ratios).

A target area whose height is equal to 10 meters and whose width is equal to 10 meters has been considered. The relative distance underground cabletarget area has been varied and the magnetic field reduction has been optimised by the GA, obtaining the results reported in Table IV and shown in figs. 3 and 4.

The GA has obviously calculated different solutions for each considered geometry (due to the different individuals that compose the evolving population), but only the solution characterized by the higher percentage of reduction of the magnetic flux density has been considered for each case.

Table III Geometrical arrangement of the considered power line

\begin{tabular}{|l|l|l|l|}
\hline Wire & $\mathrm{X}[\mathrm{m}]$ & $\mathrm{y}[\mathrm{m}]$ & Current $[\mathrm{A}]$ \\
\hline$\# 1$ & -0.25 & -1 & $450 \angle 0^{\circ}$ \\
\hline$\# 2$ & 0 & -1 & $450 \angle-120^{\circ}$ \\
\hline$\# 3$ & +0.25 & -1 & $450 \angle-240^{\circ}$ \\
\hline
\end{tabular}


86 Environmental Health Risk

Table IV Results of the optimisation

\begin{tabular}{|c|c|c|c|c|c|c|c|c|c|}
\hline \multirow[b]{2}{*}{ 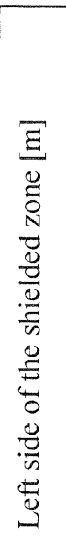 } & \multirow[b]{2}{*}{ 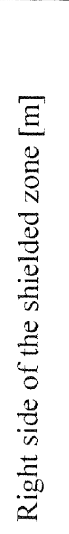 } & \multirow[b]{2}{*}{ 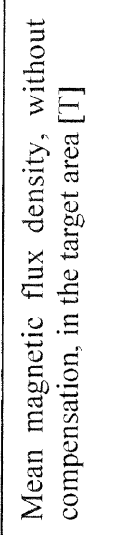 } & \multicolumn{7}{|c|}{1 or $1+1$ underground cable reduction based } \\
\hline & & & 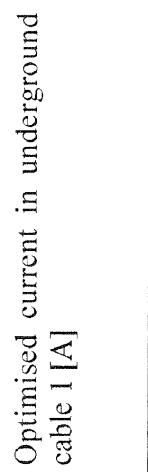 & 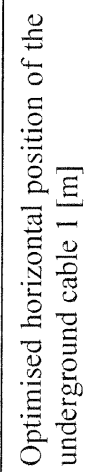 & 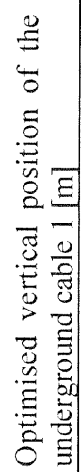 & 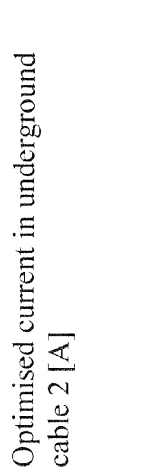 & 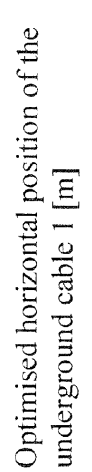 & 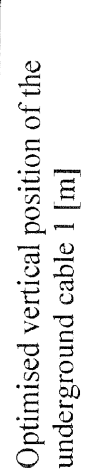 & 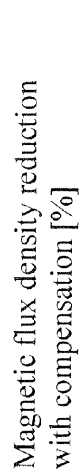 \\
\hline+5 & +15 & $3.4 \cdot 10^{-7}$ & $18 \angle 330^{\circ}$ & -0.31 & -2 & & & & 46.1 \\
\hline+5 & +15 & $3.4 \cdot 10^{-7}$ & $31.5 \angle 330$ & +1.9 & -1.1 & $31.5 \angle 150^{\circ}$ & -5.93 & -0.2 & 93.0 \\
\hline+10 & +20 & $1.6 \cdot 10^{-7}$ & $9 \angle 330^{\circ}$ & +5.62 & -2 & & & & 80.3 \\
\hline+10 & +20 & $1.6 \cdot 10^{-7}$ & $9 \angle 327^{\circ}$ & +6.87 & -0.2 & $9 \angle 147^{\circ}$ & -16.9 & 0 & 88.0 \\
\hline
\end{tabular}

Different considerations can be drawn from the obtained results.

The first consideration is that the proposed compensation method, applied to this particular underground cable arrangement, ensures significant reductions of the mean magnetic flux density that vary with the relative distance underground cable-target area, as it was expected, reaching values of the order of $93 \%$ and that are never lower than $46 \%$.

The second consideration is that the compensation cable/cables is/are always placed on the same side of the underground cable with respect to the target area. The position of the compensation cable/cables varies obviously with the relative distance underground cable-target area to reduce, as more as possible, the mean value of the magnetic flux density in the target area itself.

The third consideration is that the 1 underground cable configuration is not able to reach the elevated values $(>80 \%)$ of magnetic field reduction of the $1+1$ underground configuration when the target area is very close to the power underground cable, ensuring anyway reductions of the order of $50 \%$. When the target area is more distant, the one underground cable configuration is able to guarantee high field reductions $(>80 \%$ ) even if the $1+1$ underground configuration ensures better results in any situation with respect to it.

The last consideration is that in both the configurations ( 1 or $1+1$ cables) one cable is always placed between the power cable and the target area and the current that flows inside it tends to assume the same phase values in any configuration and position of the target area. The second cable (return current), on the contrary, is always placed on the opposite side of the target area with respect to the power cable source. 
Environmental Health Risk 87

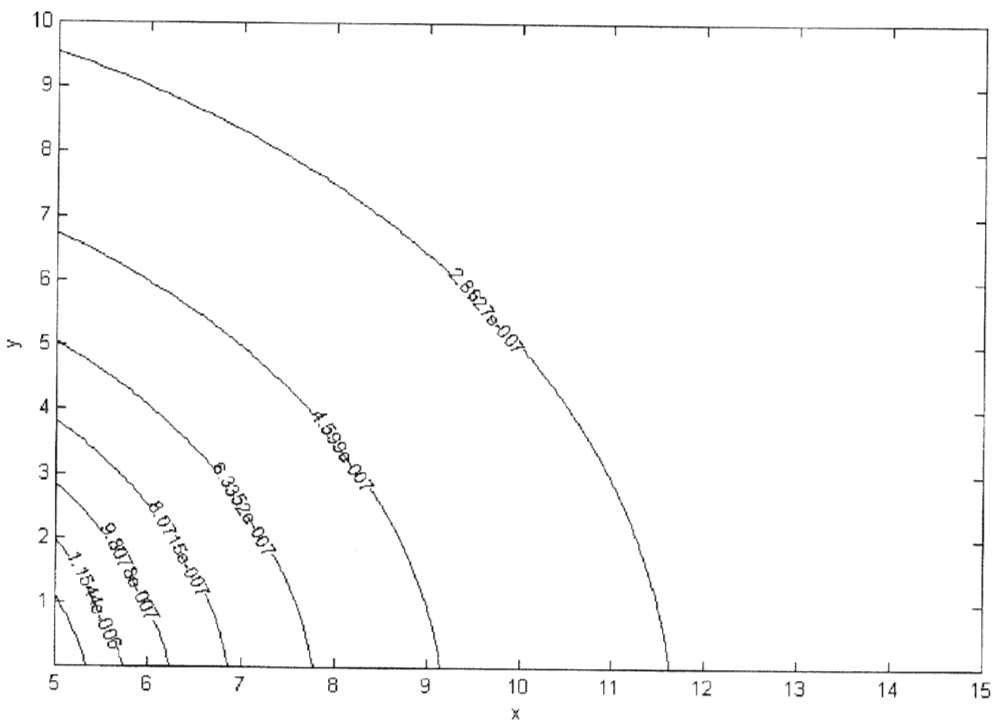

Fig. 3a

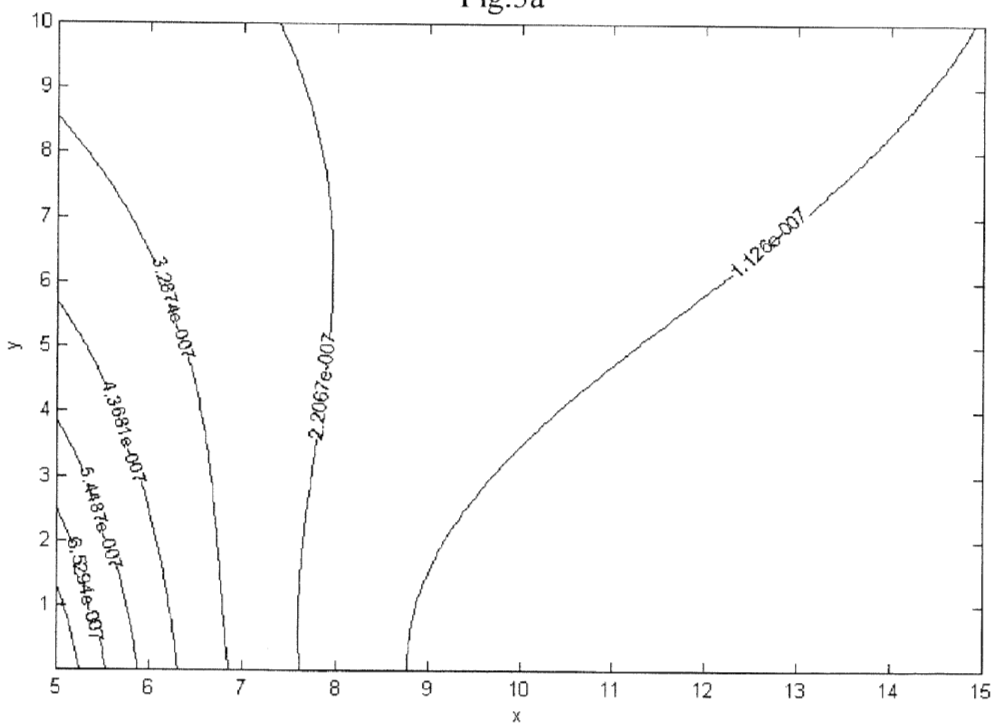

Fig. 3b

Figs.3 Magnetic flux density [T] in the considered section, (height=10 $\mathrm{m}$ ) positioned between $+5 \mathrm{~m}$ and $+15 \mathrm{~m}$ with respect to the underground cable vertical axes for different situations.

a) Magnetic field in the absence of compensation (mean magnetic flux density $=3.44 \cdot 10^{-7} \mathrm{~T}$ ).

b) 1 underground cable based compensation: magnetic field in the presence of a compensation current equal to $18 \angle 330^{\circ} \mathrm{A}$ in the underground cable positioned in $(-0.31,-2)$. The mean magnetic flux density is $46.12 \%$ lower than that in the uncompensated situation. 


\section{Environmental Health Risk}

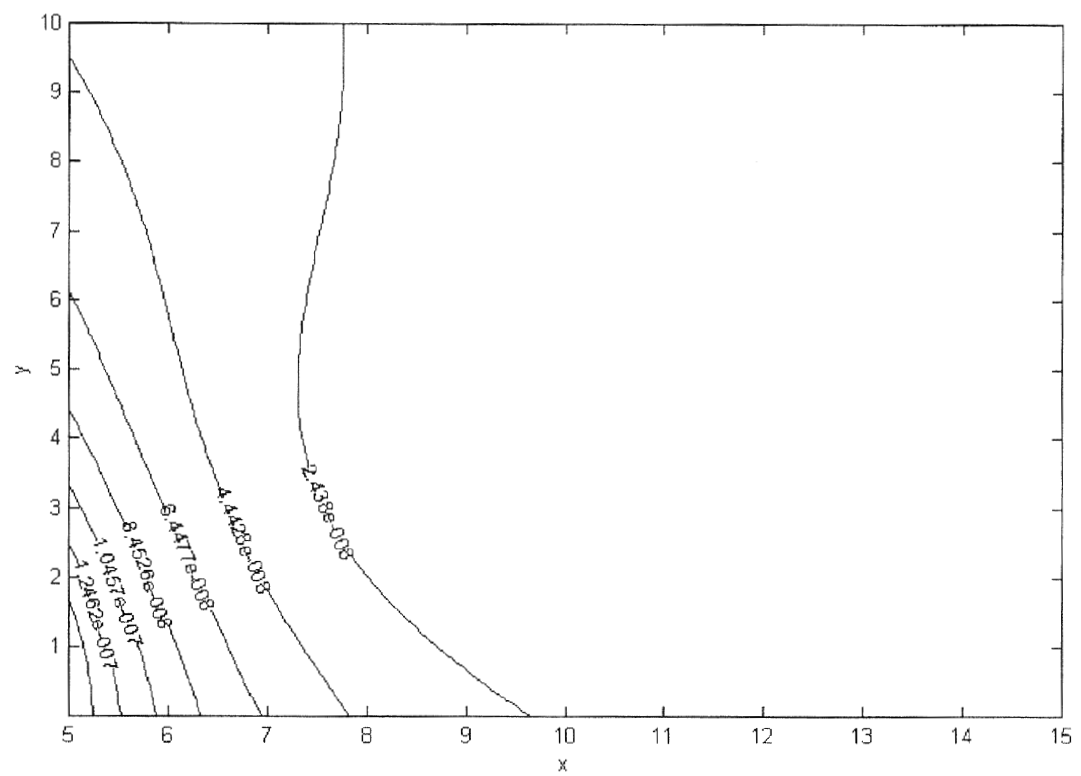

Fig. 4

Fig.4 Magnetic flux density [T] in the considered section, (height=10 $\mathrm{m}$ ) positioned between $+5 \mathrm{~m}$ and $+15 \mathrm{~m}$ with respect to the underground cable vertical axes for: $1+1$ underground cables based compensation: magnetic field in the presence of a compensation current equal to $31.5 \angle 330^{\circ} \mathrm{A}$ in the underground cable positioned in $(+1.9,-1.1)$ and a compensation current equal to $31.5 \angle 150^{\circ} \mathrm{A}$ in the underground cable positioned in $(-5.93,-0.2)$. The mean magnetic flux density is $93 \%$ lower than that in the uncompensated situation.

To verify the validity of the proposed method, different underground cables geometries and different target area configurations were optimised by the GA, obtaining generally mean magnetic flux density reduction always greater than $46 \%$ (and often much better).

\section{Conclusions}

We have presented different techniques, based on the use of active conductors for the the reduction of the magnetic field arising from either overhead lines or buried cables, which ensure significant reduction of magnetic field in target areas.

The optimal choice of the position of the compensation wires and of the currents to be driven inside them is obtained by means of genetic algorithms, which compare favourably with deterministic algorithms in terms of accuracy and computational costs.

The proposed technique can be applied to any kind of source configuration, with appropriate modifications, yielding to significant magnetic field reductions. 


\section{References}

[1] S. Celozzi, "Active compensation and partial shields for the powerfrequency magnetic field reduction", IEEE 2002 Int. Symp. on Electromagnetic Compatibility, Minneapolis, USA, pp. 222-226.

[2] S. Celozzi, F. Garzia, "Active shielding for the power-frequency magnetic field reduction using genetic algorithms optimisation", submitted for publication on IEE Proc.

[3] S. Celozzi, F. Garzia, "Hybrid active-passive shielding technique for the mitigation of power cables magnetic field", to be submitted.

[4] F. Garzia, A. Geri, "Active shielding design in a full 3D space of indoor MV/LV substations using genetic algorithms optimization", to be presented at IEEE EMC 2003, Boston.

[5] D.E. Goldberg, "Genetic algorithms in search, optimisation and machine learning", Addison Wesley, 1989. 
